DR SU M LWIN (Orcid ID : 0000-0002-3325-3675)

Received Date : 09-Jan-2017

Revised Date : 03-Mar-2017

Accepted Date : 08-Mar-2017

Article type : Case Report

\title{
Beneficial effect of ustekinumab in familial pityriasis rubra pilaris with a new missense mutation in CARD14
}

S.M. Lwin, ${ }^{1}$ C-K. Hsu, ${ }^{1,2}$ L. Liu, ${ }^{3}$ H-Y. Huang, ${ }^{2}$ N.J. Levell, ${ }^{4}$ J.A. McGrath ${ }^{1}$

${ }^{1}$ St John's Institute of Dermatology, King's College London (Guy's Campus), London, UK;

${ }^{2}$ Department of Dermatology, National Cheng Kung University Hospital, College of Medicine, National Cheng Kung University, Tainan, Taiwan; ${ }^{3}$ Viapath, St Thomas' Hospital, London, UK; ${ }^{4}$ Dermatology, Norfolk and Norwich University Hospitals NHS Foundation Trust, Norwich, UK.

Corresponding Author: John McGrath, Dermatology Research Laboratories, Floor 9 Tower Wing, Guy’s Hospital, Great Maze Pond, London SE1 9RT, UK. Tel: +44-20-71886409; Fax +44-2071888050; E-mail: john.mcgrath@kcl.ac.uk

This article has been accepted for publication and undergone full peer review but has not been through the copyediting, typesetting, pagination and proofreading process, which may lead to differences between this version and the Version of Record. Please cite this article as doi: 10.1111/bjd.15462

This article is protected by copyright. All rights reserved. 
Funding statement: The authors acknowledge financial support from the Department of Health via the UK National Institute for Health Research (NIHR) Comprehensive Biomedical Research Centre award to Guy's \& St Thomas' NHS Foundation Trust in partnership with King's College London and King's College Hospital NHS Foundation Trust.

Conflicts of interest: None declared.

Keywords: CARD14, Autosomal Dominant Pityriasis Rubra Pilaris, IL12/23 antagonist, Ustekinumab.

\section{Summary}

Pityriasis rubra pilaris (PRP) represents a group of rare chronic inflammatory skin disorders in which $\sim 1$ in 20 affected individuals show autosomal dominant inheritance. In such cases, there may be gainof-function mutations in CARD14, encoding caspase recruitment domain-containing protein 14 (CARD14) that activates the non-canonical nuclear factor-kappa B (NF- $\kappa \mathrm{B})$ pathway, thereby promoting cutaneous inflammation. Here, we report a mother and son with PRP due to a new missense mutation in CARD14 and describe the beneficial clinical effects of ustekinumab, a monoclonal antibody against interleukins-12 and -23 , in both subjects. A 49 year-old female and her 20 year-old son had lifelong, generalised, patchy erythematous scale with a few islands of sparing, as well as minor nail ridging and mild palmoplantar keratoderma, features consistent with generalised PRP. Topical steroids, phototherapy and oral retinoids proved ineffective therapies. Following informed consent, Sanger sequencing of CARD14 in both individuals revealed a new heterozygous single nucleotide transversion in exon 16 , c.356T $>\mathrm{G}$, resulting in the missense mutation, p.Met119Arg. Ustekinumab, at a dose of $45 \mathrm{mg}$ every 12 weeks, brought about a significant physical and emotional improvement in both the mother and son within a few days of the initial dose, which

This article is protected by copyright. All rights reserved. 
was sustained on maintenance dosing. This report highlights the therapeutic potential of biologics that downregulate NF-kB signalling in familial PRP with mutations in CARD14.

\section{What's already known about this topic?}

- Pityriasis rubra pilaris (PRP) represents a group of rare chronic inflammatory skin disorders in which some cases show autosomal dominant inheritance; some cases also reveal heterozygous gain-of-function mutations in CARD14, encoding caspase recruitment domain-containing protein 14 (CARD14).

- Ustekinumab, an IL12/23 antagonist, is a licensed biological therapy for psoriasis, although its use has not been widely assessed in PRP, despite anecdotal reports of its potential efficacy.

\section{What does this study add?}

- We report a mother and son with refractory generalised PRP. DNA sequencing revealed they both carried a new de novo missense mutation in CARD14, p.Met119Arg.

- Identification of this CARD14 mutation was used as justification for a trial of ustekinemab, whereupon both subjects had a beneficial clinical response, physically and emotionally, with sustained improvements over several months of follow up.

- Ustekinumab may be considered as a targeted anti-inflammatory therapy for familial PRP with an underlying mutation in CARD14.

Pityriasis rubra pilaris (PRP) represents a group of rare chronic inflammatory skin disorders, which may show clinico-pathological overlap with psoriasis. ${ }^{1,2}$ Most cases of PRP are sporadic but $~ 1$ in 20 show autosomal dominant inheritance. ${ }^{3}$ Moreover, some familial PRP has been shown to result from gain-of-function mutations in CARD14, encoding caspase recruitment domain-containing

This article is protected by copyright. All rights reserved. 
protein 14 (CARD14). ${ }^{1}$ CARD14, also known as CARD-containing membrane-associated guanylate kinase (MAGUK) protein 2 (Carma 2), is a member of a family of scaffold proteins involved in cell adhesion, signal transduction and cell polarity control. ${ }^{4}$ CARD14 binds to B cell lymphoma 10 (BCL10) through a CARD-CARD interaction and activates the non-canonical nuclear factor-kappa B (NF- $\mathrm{\kappa B})$ pathway, thus promoting inflammation. ${ }^{4}$

The significance of CARD14 mutations in PRP may also extend to therapy since a few previous reports have highlighted good clinical responses to biologics, such as ustekinumab, when conventional anti-inflammatory therapies have not led to patient benefit. ${ }^{5-8}$ Of all the reports, however, the benefit of ustekinumab was demonstrated in only one case with a known CARD14 mutation. ${ }^{8}$ In this report, we describe a mother and son with generalised PRP, identify a new CARD14 mutation, and demonstrate major physical and emotional improvements following treatment with ustekinumab.

\section{Case Report}

A 49 year-old female presented with widespread erythema and scale. Her skin had been normal at birth but around aged six weeks she developed erythematous patches on both cheeks followed by more generalised dry red scales with peeling that persisted into adulthood. Examination revealed generalised, confluent erythematous scale affecting the face, body and limbs, and a few islands of spared, apparently unaffected skin. She had minor nail ridging and mild palmoplantar keratoderma. Her 20 year-old son had a similar clinical course that began in his first few weeks of life although his skin developed more patchy, serpiginous, dry and thick scale with associated erythema. A clinical diagnosis of autosomal dominant familial PRP was made (pedigree is shown in Fig. 1a). Treatment with oral retinoids, phototherapy, and topical steroids proved ineffective in both subjects.

Following informed consent, Sanger sequencing of CARD14 using peripheral blood DNA as template, was performed on both affected and all unaffected members of the family. This revealed a heterozygous single nucleotide transversion c.356T $>\mathrm{G}$ in exon 16 , which converts methionine (ATG)

This article is protected by copyright. All rights reserved. 
to arginine (AGG), designated p.Met119Arg (Fig 1b). This missense mutation was detected in both the affected mother and her son but not in other unaffected relatives nor in $>120,000$ control alleles (http://exac.broadinstitute.org/gene/ENSG00000141527). This amino acid substitution is located adjacent to other gain-of-function mutations between the CARD and coiled-coil domain. ${ }^{1,9}$ The functional effect of this missense mutation, assessed with various programs, ${ }^{10-14}$ strongly indicates its pathogenic potential that p.Met119Arg is a pathologic variant (SIFT: 0.107, tolerated; PolyPhen-2: 0.244, benign; MutationTaster: 0.999, disease causing; CADD: 23.9, damaging; DANN: 0.964, pathogenic).

Both mother (weight $=66 \mathrm{~kg}$ ) and son (weight $=65 \mathrm{~kg}$ ) were treated with sub-cutaneous ustekinumab 45mg every 12 weeks after two loading doses at weeks 0 and 4 . Both Psoriasis Area Severity Index (PASI) - adopted as a measure of disease severity for PRP, and Dermatology Quality of Life Index (DLQI) were assessed for the mother and son at baseline, 12 weeks and 24 weeks after the initiation of ustekinumab. At baseline, the mother had a PASI of 25.7 and DLQI 18, and the son had a PASI of 29.2 and DLQI 22. A dramatic clinical improvement was observed within a few days of the initial loading dose and was clearly evident after 12 weeks with corresponding PASI $<1$ and DLQI 0 for the mother, and PASI 4.8 and DLQI 3 for the son (Fig. 1c), which persisted for a total of 24 weeks. The clinical benefit in the mother, however, waned around 30 weeks after initiation, but the skin improved again after the dose of ustekinumab was increased from $45 \mathrm{mg}$ to $90 \mathrm{mg} 12$-weekly. Approximately 12 weeks after the ustekinumab dose increase, the mother had normal fingernails for the first time in her life. Both individuals have been observed for $>8$ months on treatment and the improvement has been maintained. For individuals with psoriasis, an HLA-Cw6 genotype has been associated with an improved response to ustekinumab but genotyping in both mother and son with PRP (using published methods, ref. 15) revealed that they were both HLA-Cw6-negative. There were noticeable personal benefits for both individuals, due to improved physical and emotional wellbeing, following the improvement with ustekinumab after many years of impaired quality of life due to PRP. Details of the positive impact the ustekinumab had on the mother's life are recorded in her own words in the Supplementary material.

This article is protected by copyright. All rights reserved. 
In summary, the positive response to treatment with ustekinumab in both the mother and son highlights the efficacy of this biologic therapy in PRP, with the discovery of an underlying mutation in CARD14 being the driver towards pursuing this particular targeted anti-inflammatory therapy.

\section{Acknowledgement}

We thank the Norfolk and Norwich University Hospital Medical Illustration Department and St John's Institute of Dermatology Medical Illustration Department for clinical images.

\section{References}

1. Fuchs-Telem D, Sarig O, van Steensel MAM et al. Familial pityriasis rubra pilaris is caused by mutations in CARD14. Am J Hum Genet 2012; 91:163-170.

2. Jordan CT, Cao L, Roberson EDO et al. Rare and common variants in CARD14, encoding an epidermal regulator of NF-kappaB, in psoriasis. Am J Hum Genet 2012; 90:784-95.

3. Albert MR, Mackool BT. Pityriasis rubra pilaris. Int J Dermatol 1999; 38:1-11.

4. Bertin J, Wang L, Guo Y et al. CARD11 and CARD14 are novel caspase recruitment domain (CARD)/membrane-associated guanylate kinase (MAGUK) family members that interact with BCL10 and activate NF-kappa B. J Biol Chem 2001; 276:11877-82.

5. Ruiz Villaverde R, Sanchez Cano D. Successful treatment of type 1 pityriasis rubra pilaris with ustekinumab therapy. Eur J Dermatol 2010; 20:630-1.

6. Wohlrab J, Kreft B. Treatment of pityriasis rubra pilaris with usetkinumab. Br J Dermatol 2010; 163:655-6.

7. Eastham A, Femia AN, Qureshi A et al. Treatment options for pityriasis rubra pilaris including biologic agents: a retrospective analysis from an academic medical center. JAMA Dermatol 2014; 150:92-4.

This article is protected by copyright. All rights reserved. 
8. Eytan O, Sarig O, Sprecher E et al. Clinical response to ustekinumab in familial pityriasis rubra pilaris caused by a novel mutation in CARD14. Br J Dermatol 2014; 171:420-22.

9. Takeichi T, Sugiura K, Nomura $\mathrm{T}$ et al. Pityriasis rubra pilaris type $\mathrm{V}$ as an autoinflammatory disease by CARD14 mutations. JAMA Dermatol 2016; 153:66-70.

10. Kumar P, Henikoff S, Ng PC. Predicting the effects of coding non-synonymous variants on protein function using the SIFT algorithm. Nat Protoc 2009; 4:1073-81.

11. Adzhubei I, Jordan DM, Sunyaev SR. Predicting functional effect of human missense mutations using PolyPhen-2. Curr Protoc Hum Genet 2013; Chapter 7: Unit 720.

12. Schwarz JM, Rodelsperger C, Schuelke $M$ et al. MutationTaster evaluates disease-causing potential of sequence alterations. Nat Methods 2010; 7:575-6.

13. Kircher $\mathrm{M}$, Witten $\mathrm{DM}$, Jain $\mathrm{P}$ et al. A general framework for estimating the relative pathogenicity of human genetic variants. Nat Genet 2014; 46:310-5.

14. Quang D, Chen Y, Xie X. DANN: a deep learning approach for annotating the pathogenicity of genetic variants. Bioinformatics 2015; 31:761-3.

15. Caldarola G, Sgambato A, Caterina $F$ et al. HLA-Cw6 allele, NFkB1 and NFkB1A polymorphisms play no role in predicting response to etanercept in psoriatic patients. Pharmacogenet Genomics 2016; 26:423-7.

This article is protected by copyright. All rights reserved. 


\section{Figure Legends}

Fig. 1. Pedigree, molecular pathology and clinical features of the mother and son with autosomal dominant pityriasis rubra pilaris.

(a) Pedigree: Sanger sequencing of CARD14 was performed in all family members (affected and unaffected) shown in this autosomal dominant pedigree (mother is indicated by arrow); (b) Heterozygous missense mutation (c.356T>G; p.Met119Arg) in CARD14, which was present in the affected mother and son but none of the other unaffected family members; (c) Clinical responses at baseline and after 12 weeks therapy with ustekinumab in the mother and son.

\section{Supplementary Material}

A personal account by the mother with familial PRP on "how ustekinumab has changed (her) life".

This article is protected by copyright. All rights reserved. 


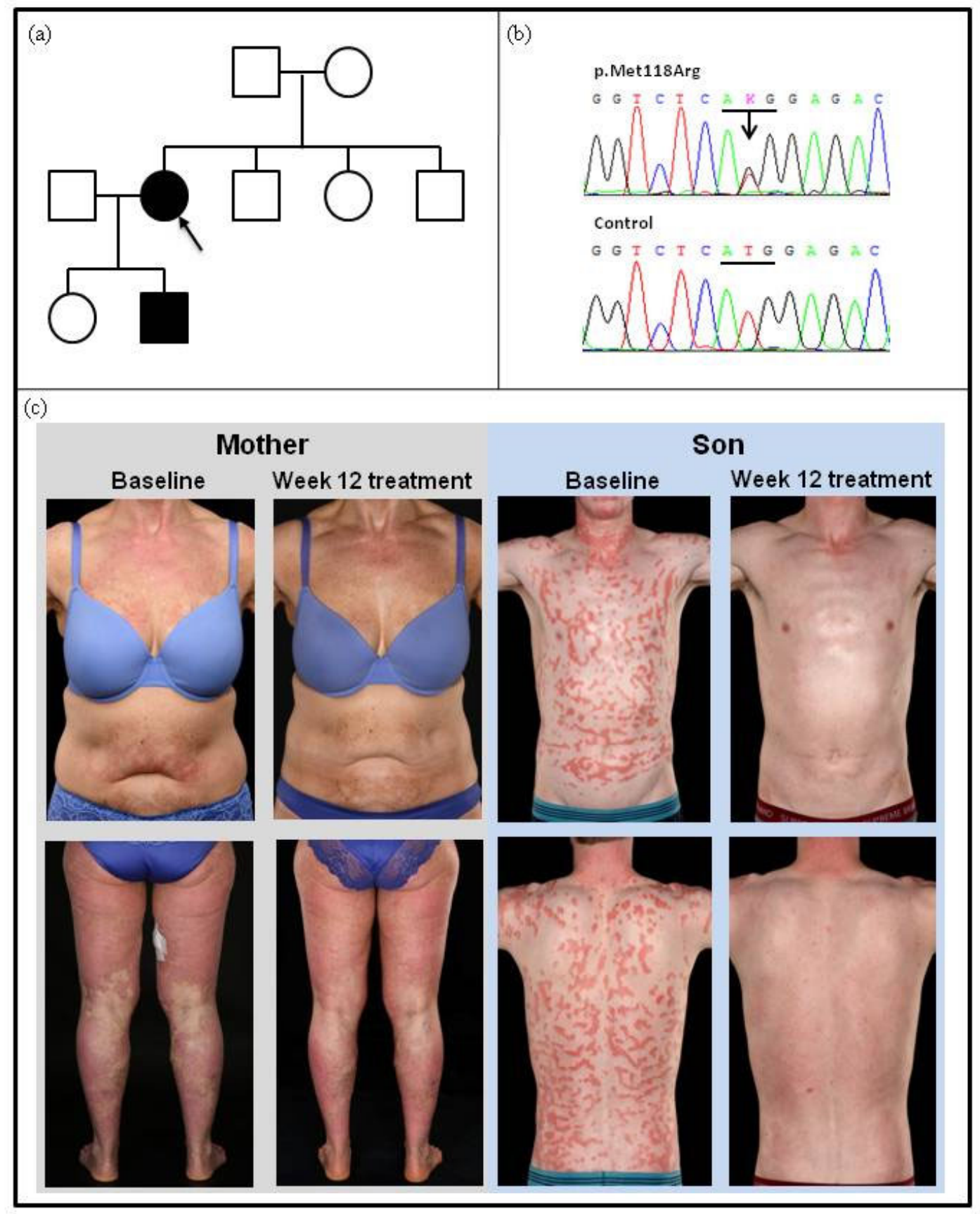

This article is protected by copyright. All rights reserved. 